\title{
Effect of epidural analgesia in trial of labor after cesarean on maternal and neonatal outcomes in China: a multicenter, prospective cohort study
}

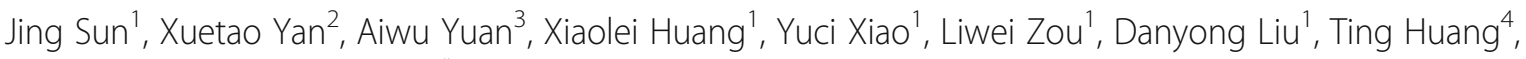
Zhao Zheng ${ }^{1}$ and Yuantao $\mathrm{Li}^{1^{*}}$

\begin{abstract}
Background: The trial of labor after cesarean section (TOLAC) is a relatively new technique in mainland of China, and epidural analgesia is one of the risk factors for uterine rupture. This study aimed to evaluate the effect of epidural analgesia on primary labor outcome [success rate of vaginal birth after cesarean (VBAC)], parturient complications and neonatal outcomes after TOLAC in Chinese multiparas based on a strictly uniform TOLAC indication, management and epidural protocol.
\end{abstract}

Methods: A total of 423 multiparas undergoing TOLAC were enrolled in this study from January 2017 to February 2018. Multiparas were divided into two groups according to whether they received epidural analgesia (study group, $N=263$ ) or not (control group, $N=160$ ) during labor. Maternal delivery outcomes and neonatal characteristics were recorded and evaluated using univariate analysis, multivariable logistic regression and propensity score matching (PSM).

Results: The success rate of VBAC was remarkably higher $(85.55 \%$ vs. $69.38 \%, p<0.01)$ in study group. Epidural analgesia significantly shortened initiating lactation period and declined Visual Analogue Score (VAS). It also showed more superiority in neonatal umbilical arterial blood pH value. After matching by PSM, multivariable logistic regression revealed that the correction of confounding factors including epidural analgesia, cervical Bishop score at admission and spontaneous onset of labor were still shown as promotion probability in study group (OR $=4.480$, 1.360 , and 10.188 , respectively; $95 \% \mathrm{Cl}=2.025-10.660,1.113-1.673$, and $2.875-48.418$, respectively; $p<0.001, p=$ 0.003 , and $p<0.001$, respectively).

Conclusions: Epidural analgesia could reduce labor pain, and no increased risk of postpartum bleeding or uterine rupture, as well as adverse effects in newborns were observed. The labor duration of multiparas was increased, but within acceptable range. In summary, epidural analgesia may be safe for both mother and neonate in the three studied hospitals.

Trial registration: Chineses Clinical Trial Register, ChiCTR-ONC-17010654. Registered February 16th, 2017.

Keywords: Epidural analgesia, Trial of labor after cesarean, Vaginal birth after cesarean, Maternal and neonatal outcomes, Propensity score matching

\footnotetext{
* Correspondence: Liyuantao6788@yeah.net

'Department of Anesthesiology, Affiliated Shenzhen Maternity \& Child Healthcare Hospital, Southern Medical University, No.2004 Hongli Road, Futian District, Shenzhen 518028, Guangdong, China

Full list of author information is available at the end of the article
}

(c) The Author(s). 2019 Open Access This article is distributed under the terms of the Creative Commons Attribution 4.0 International License (http://creativecommons.org/licenses/by/4.0/) which permits unrestricted use, distribution, and reproduction in any medium, provided you give appropriate credit to the original author(s) and the source, provide a link to the Creative Commons license, and indicate if changes were made. The Creative Commons Public Domain Dedication waiver (http://creativecommons.org/publicdomain/zero/1.0/) applies to the data made available in this article, unless otherwise stated. 


\section{Background}

The proportion of pregnant women undergoing cesarean section has augmented steadily in the past few decades and reached the highest in the world, especially in China $[1,2]$. Cesarean section can reduce maternal and neonatal mortality, but overuse may result in severe maternal outcomes, such as enhanced risk of death [3]. At present, delivery modes for multiparas receive increasing concerns with the implementation of the two-child policy in China. After the first cesarean section, the alternative mode of subsequent labor includes repeated cesarean section (RCS) and the trial of labor after cesarean (TOLAC). Recent data show that TOLAC is the most effective method of delivery because of the less expense and better effect in reducing the risk of postpartum hemorrhage $(\mathrm{PPH})$ and pelvic adhesions [4]. In addition, several investigators had attempted to create formulae to calculate individual specific TOLAC results. Costantine MM et al. [5] established a predictive model for a cohort of women with a single previous cesarean section, and found that the success rate of vaginal birth after cesarean (VBAC) was 10-20\% lower than that of women who predicted $>50 \% \mathrm{VBAC}$ success rate. Grobman WA et al. [6] also created a nomogram using factors available at the first prenatal visit and developed a useful tool to measure patient-specific VBAC success rate. These studies indicate that multiparas with an expected success rate of $>50 \%$ are appropriate candidates for TOLAC, which needs to be clinically validated.

Epidural analgesia is a pre-requisite for many women to choose TOLAC, and for obstetricians, it is a means of allowing adequate and long-lasting pain relief in the management of normal and dysfunctional labors. However, none of above predictive models has mentioned analgesic effect. TOLAC is still in the early stages in mainland of China because of its unique medical environment. Notably, the doctor-patient relationship in China is tense due to patient distrust. Therefore, in order to reduce medical disputes, doctors often have to choose the surgical treatment according to patients' needs, rather than the natural childbirth based on patients' own conditions [7]. With increasing international acceptance of TOLAC and improved technology, more and more Chinese medical institutions begin to use TOLAC. It has been reported that the epidural analgesia is unsafe for maternal and newborn at risk of uterine rupture, but the 2010 American Association of Obstetricians and Gynecologists (ACOG) guidelines explicitly recommend that epidural anesthesia in TOLAC is safe $[8,9]$. In addition, different policies for labor dystocia management and epidural anesthesia permissiveness at TOLAC challenge the interpretation of previous studies. Several reports have revealed the relationship between labor pain intensity and dystocia, suggesting that obstructed labor rate is higher as labor pain increased, while pain relief makes labor go smoothly [10-12]. A correlation was shown of endogenous plasma epinephrine and cortisol levels with labor progression $[13,14]$. Neumark J et al. [15] have demonstrated that cortisol level significantly rises in women undergoing epidural analgesia, while decreases as epinephrine level declined after pain relief. Besides, the decrease in alphaand beta-adrenergic stimulus may enhance uterine perfusion and further lead to an effective uterine contraction pattern, since its sensitivity is characteristic of the uteroplacental vascular bed rather than the systemic vasculature [16]. Therefore, we hypothesize that epidural analgesia reduces maternal epinephrine levels by eliminating psychological and physical stress associated with painful uterine contractions, thus promotes delivery. This brings more opportunities for TOLAC and emphasizes the low rate of side effects of epidural anesthesia for both mother and fetus [17].

At present, epidural analgesia has not been widely used in TOLAC due to controversy. Domestic and foreign studies on the application of epidural analgesia in TOLAC are mostly small samples or retrospective studies, but lack of large sample investigation or systematic study. This multi-center study aimed to evaluate the effect of epidural analgesia on primary labor outcome (success rate of VBAC), parturient complications and neonatal outcomes after TOLAC in Chinese multiparas based on a strictly uniform TOLAC indication, management, and epidural protocol.

\section{Methods}

\section{Ethics and informed consent}

This multi-center, prospective cohort study was approved by the human research committee of Affiliated Shenzhen Maternity \& Child Healthcare Hospital, Bao'an Maternal and Child Health Hospital, and Longgang District Maternity \& Child Healthcare Hospital of Shenzhen City, and written informed consents were obtained from all subjects participating in the trial. The study was registered prior to patient enrollment at the Chinese Clinical Trial Register, a participant in the World Health Organization International Clinical Trials Registry Platform (ChiCTR-ONC17010654, Principal investigator: Jing Sun, Date of registration: February 16th, 2017).

\section{Study design and multiparas' recruitment}

Totally 443 multiparas undergoing TOLAC were enrolled in this study from January 2017 to February 2018. They were divided into two groups according to their own requests of epidural analgesia, i.e., multiparas who received epidural analgesia were in study group; while matched multiparas who did not receive epidural analgesia were in control group. After excluding 20 follow- 
up misses, a total of 423 multiparas, including 263 in study group and 160 in control group, were entered into the final analysis (Fig. 1), and the baseline maternal demographic and obstetric characteristics were recorded (Table 1). Inclusion criteria: multiparas with a single live fetus in cephalic presentation at $>35$ weeks; no systemic analgesics were currently used; eligible for TOLAC under department admission and management protocol. The eligibility criteria for TOLAC (as assessed by obstetricians) were as follows: a. willingness of undergoing TOLAC and acceptance of the possible risks; b. good health condition without contraindications of vaginal delivery; c. immediate access to emergency surgery; $d$. confirmation of successful history of cesarean section with low-transverse segment, no delayed incision, scheduled recovery, as well as no late postpartum hemorrhage and postpartum infection; e. more than 1 year from last cesarean section; f. no history of uterine rupture; g. fetal weight $<4200 \mathrm{~g}$ (estimated through clinical assessment or ultrasound exam within a week from admission). TOLAC can be performed regardless of the number of uterine closure layers in cesarean section at the first time, and a diagnosis of dystocia of labor for previous cesarean is not considered as a contraindication for TOLAC. However, multiparas with a history of mental illness and contraindications to epidural labor analgesia were excluded from the study. All eligible women were informed of the study and signed a consent form when they entered Labor Analgesia Consultation Clinic during 34-35 weeks prenatal. However, it was also possible for a multipara to change her mind when she entered the delivery room and began delivery.

\section{The method of analgesia}

All of the investigated multiparas were observed in the delivery room, and initial laboratory evaluation was performed including blood type, hemoglobin, complete platelet count and coagulation function. They underwent continuous electronic fetal monitoring throughout delivery and were carefully evaluated to assess TOLAC's suitability. Any of the following indications should be noted when an immediate emergency cesarean section was happened, including cessation of labor, abnormal fetal heart rates (FHRs) and suspected uterine rupture. Multiparas admitted for TOLAC were managed by certified midwives who determined eligibility for TOLAC, induction of labor or oxytocin augmentation, mode of delivery, use of episiotomy, forceps and vacuum extraction for operative vaginal delivery in accordance with maternal and fetal indications made by board certified obstetricians. The multiparas participating in the study did not experience changes in obstetric clinical management during delivery.

After entering the delivery room, multiparas in the study group were placed in the left lateral position with opened peripheral venous access. Then, epidural

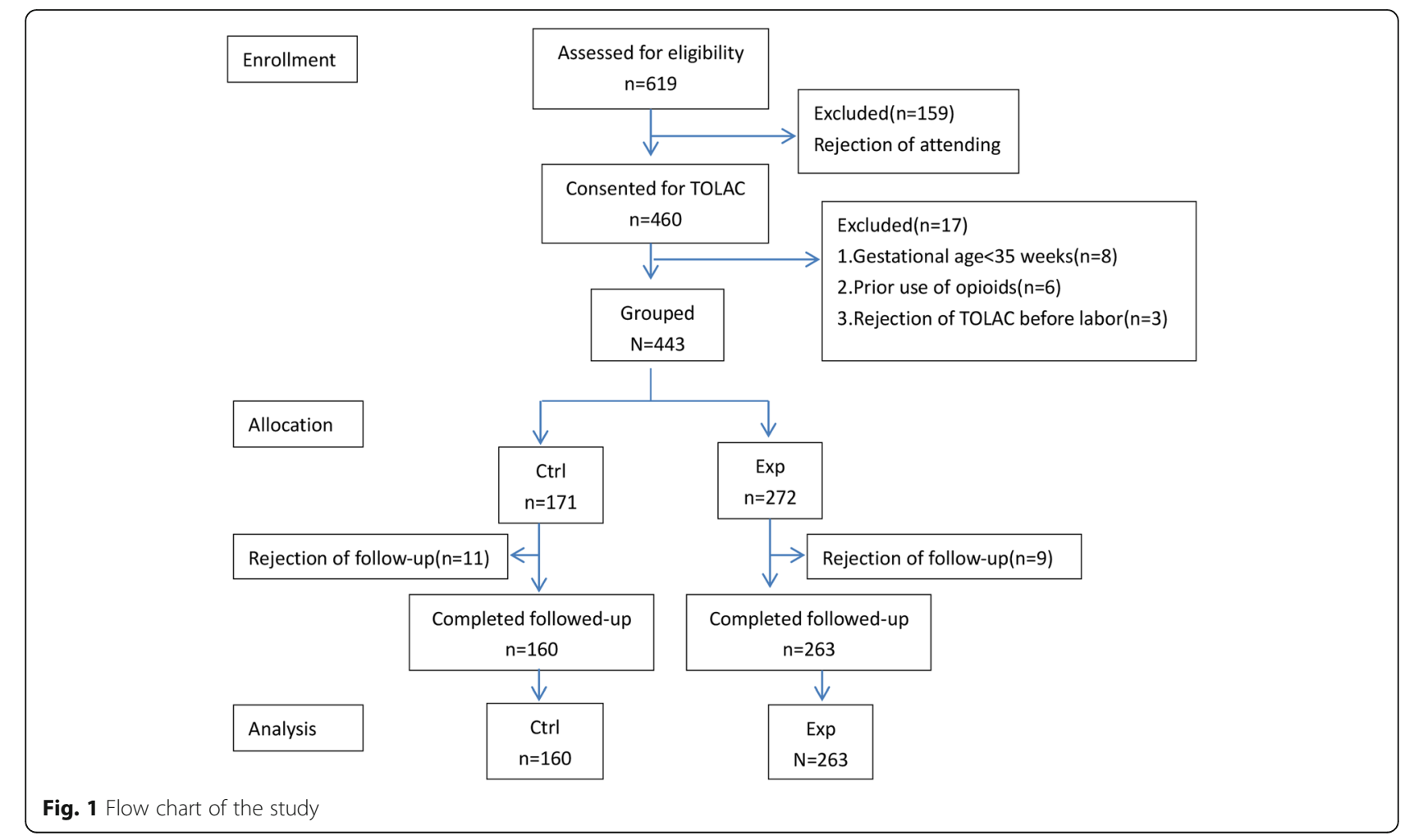


Table 1 Baseline Maternal Demographic and Obstetric Characteristics

\begin{tabular}{|c|c|c|c|c|c|c|}
\hline \multirow[t]{2}{*}{ Characteristic } & \multicolumn{2}{|c|}{ TOLAC (control) } & \multicolumn{2}{|c|}{ TOLAC (study) } & \multirow[t]{2}{*}{ Statistics } & \multirow[t]{2}{*}{$P$ value } \\
\hline & $\begin{array}{l}\mathrm{N} \\
(\text { All })\end{array}$ & $\begin{array}{l}N(\%) \text { or } \\
\text { Mean } \pm \text { SD }\end{array}$ & $\begin{array}{l}\mathrm{N} \\
\text { (All) }\end{array}$ & $\begin{array}{l}\mathrm{N}(\%) \text { or } \\
\text { Mean } \pm \mathrm{SD}\end{array}$ & & \\
\hline Hospital name & 160 & & 263 & & 41.02053 & $<0.001$ \\
\hline $\mathrm{MCH}$ & & $131(81.88 \%)$ & & $179(68.06 \%)$ & & \\
\hline BA & & $1(0.62 \%)$ & & $59(22.43 \%)$ & & \\
\hline LG & & $28(17.50 \%)$ & & $25(9.51 \%)$ & & \\
\hline \multicolumn{7}{|l|}{ General information } \\
\hline Age $(y)$ & 160 & $32 \pm 4$ & 262 & $32 \pm 4$ & $21,481.5$ & 0.667 \\
\hline Gestational age (day) & 158 & $272 \pm 14$ & 260 & $274 \pm 10$ & $18,846.5$ & 0.157 \\
\hline BMI $\left(\mathrm{kg} / \mathrm{m}^{2}\right)$ & 158 & $26.45 \pm 2.93$ & 259 & $26.59 \pm 3.08$ & $19,040.5$ & 0.234 \\
\hline Maternal education $>12 \mathrm{y}$ & 116 & $116(72.96 \%)$ & 193 & 193(74.23\%) & & 0.819 \\
\hline Husband education $>12$ y & 118 & $118(74.21 \%)$ & 207 & $207(79.62 \%)$ & & 0.228 \\
\hline Housewives & 159 & 13(8.18\%) & 259 & $21(8.11 \%)$ & & 1 \\
\hline \multicolumn{7}{|l|}{ History of pregnancy and childbirth } \\
\hline History of previous vaginal delivery & 151 & $14(9.27 \%)$ & 254 & 15(5.91\%) & & 0.233 \\
\hline Gravidity & 157 & & 260 & & 2.264 & 0.322 \\
\hline 2 & & $70(44.59 \%)$ & & $98(37.69 \%)$ & & \\
\hline 3 & & $62(39.49 \%)$ & & $121(46.54 \%)$ & & \\
\hline$>3$ & & $25(15.92 \%)$ & & $41(15.77 \%)$ & & \\
\hline Time from last cesarean section (y) & 156 & & 261 & & & 0.042 \\
\hline$\leq 3$ & & $1(0.64 \%)$ & & $9(3.45 \%)$ & & \\
\hline$\leq 5$ & & $88(56.41 \%)$ & & $165(63.22 \%)$ & & \\
\hline$>5$ & & $67(42.95 \%)$ & & $87(33.33 \%)$ & & \\
\hline $\begin{array}{l}\text { Progress of Labor during previous } \\
\text { Caesarean section (cervical dilation: } \mathrm{cm} \text { ) }\end{array}$ & 148 & & 256 & & 5.093 & 0.165 \\
\hline 0 & & $83(56.08 \%)$ & & $130(50.78 \%)$ & & \\
\hline$<3$ & & $36(24.32 \%)$ & & $87(33.98 \%)$ & & \\
\hline$\geq 3$ & & 19(12.84\%) & & $29(11.33 \%)$ & & \\
\hline 10 & & 10(6.76\%) & & 10(3.91\%) & & \\
\hline Cervical score at admission (score) & 151 & $6.54 \pm 1.76$ & 253 & $6.22 \pm 2.12$ & $20,321.5$ & 0.27 \\
\hline Cervical dilation at admission $<3 \mathrm{~cm}$ & 156 & $108(69.23 \%)$ & 259 & $237(91.51 \%)$ & & $<0.001$ \\
\hline Effacement $\leq 50$ & 151 & $47(31.13 \%)$ & 256 & $80(31.25 \%)$ & & 1 \\
\hline The onset of labor: Spontaneous & 156 & $136(87.18 \%)$ & 262 & $251(95.80 \%)$ & & 0.002 \\
\hline Oxytocin during labor & 144 & 10(6.94\%) & 244 & $22(9.02 \%)$ & & 0.568 \\
\hline \multicolumn{7}{|l|}{ Maternal comorbidities } \\
\hline Gestational diabetes mellitus & 155 & $16(10.32 \%)$ & 261 & 23(8.81\%) & & 0.606 \\
\hline Hypertensive disorders of pregnancy & 155 & $3(1.94 \%)$ & 260 & $4(1.54 \%)$ & & 0.716 \\
\hline Hypothyroidism & 155 & $4(2.58 \%)$ & 260 & $8(3.08 \%)$ & & 1 \\
\hline Asthma & 153 & $0(0.00 \%)$ & 261 & $3(1.15 \%)$ & & 0.299 \\
\hline \multicolumn{7}{|l|}{ Prepartum Laboratory test } \\
\hline HGB (g/L:115-150) & 159 & $119.28 \pm 15.05$ & 259 & $116.68 \pm 11.17$ & 22,643 & 0.087 \\
\hline PLT (125-350) & 159 & $209.47 \pm 55.51$ & 260 & $210.03 \pm 53.70$ & $20,399.5$ & 0.822 \\
\hline
\end{tabular}

$B M I$ Body mass index, HGB Hemoglobin, PLT Platelet Data are Mean \pm SD, or N (\%). SD Standard deviation Comparisons were made using two-sided Student's $t$ test or Wilcoxon rank sum test for non-normally distributed variables Comparisons were made using Pearson's Chi-squared test and Fisher's exact test for proportions 
puncture (AS-E epidural puncture package) was performed through L2-L3 or L3-L4 intervals, and the epidural catheter was inserted at a depth of $3-4 \mathrm{~cm}$. Then, an experimental dose (a total of $3 \mathrm{~mL}$ of 1:200,000 adrenalin $+1.5 \%$ lidocaine) was injected. If there was no local anesthetic poisoning or other abnormal reactions, the catheter was fixed and the multiparas were placed in the supine position. For pain relief, $10 \mathrm{~mL}$ of $0.1 \%$ ropivacaine mixed solution was injected once through the epidural catheter. If there were no obvious adverse reactions such as hypotension, nausea and vomiting or local anesthetic poisoning symptoms after $30 \mathrm{~min}$ of observation, an analgesia pump (ZZB-I impulse type, $200 \mathrm{~mL}$ ) was connected, with a ready to use solution of $0.08 \%$ ropivacaine and sufentanil $0.4 \mu \mathrm{g} / \mathrm{ml}$. Parameter setting: pulse frequency once/h, dose $10 \mathrm{~mL}$, infusion rate 400 $\mathrm{ml} / \mathrm{h}$, PCA dose $8 \mathrm{ml}$, locking time $30 \mathrm{~min}$. Epidural labor analgesia continued until the baby was delivered. Multiparas could terminal their epidural infusion at the request of the obstetric care provider for clinical indications. There was no standardization of the indication for termination nor was there a specific requirement other than obstetric request. Blood pressure was measured every $5 \mathrm{~min}$ during the first $20 \mathrm{~min}$ and hourly during the continuous of patient-controlled analgesia usage [18]. Meanwhile, Visual analog scale (VAS) pain scores was recorded $30 \mathrm{~min}$ after the epidural loading dose.

\section{Observational index}

The primary outcome was the success rate of VBAC. Maternal and neonatal parameters were recorded. The maternal parameters included demographic characteristics, mode of delivery, reason for cesarean, uterine rupture, postpartum hemorrhage (a total blood loss $>500$ $\mathrm{ml}$ within $24 \mathrm{~h}$ ), labor duration, initiating lactation period (from fetal delivery to maternal conscious breast swelling, and the milk overflow on light pressure), and VAS score. VAS score was used to assess the pain level which presented a $10-\mathrm{cm}$ unmarked line with endpoints labeled "no pain" and "worst pain imaginable" to let patients mark their pain level on [19].

Neonatal parameters were assessed by Apgar score at $1 \mathrm{~min}$ and $5 \mathrm{~min}$, birth weight, umbilical arterial blood $\mathrm{pH}$ value and fetal distress (category III FHR tracing, meconium amniotic fluid with an abnormal FHR, or an umbilical cord blood $\mathrm{pH}<7.2$ ).

\section{Statistical analysis}

All data were entered and analyzed in R software (version 3.5.0). Continuous data were expressed as Mean \pm standard deviation $(\mathrm{x} \pm \mathrm{s})$ and $t$-test was used for comparison between groups. Measurement data not conforming to normal distribution were compared using two-sided Student's T test or Wilcoxon rank sum test.
Categorical variables were presented as frequencies and percentages; Fisher's exact test or Pearson's Chi-squared test was used to examine difference between the groups. The Shapiro-Wilk test was used to assess the normal distribution of the data. Univariate analysis was used to analyze all risk factors that may affect outcomes of this study. Multivariable logistic regression models were adjusted to examine the effect of epidural analgesia on outcomes in TOLAC. Propensity score matching (PSM) were used in this study to reduce the potential selection bias and validate the results again. $P$ value $<0.05$ was considered the statistical significance.

\section{Results}

\section{Baseline maternal demographic and obstetric} characteristics

The study flow chart was shown in Fig. 1. Totally 423 multiparas enrolled into the final analysis were divided into two groups according to whether they received epidural analgesia (study group, $n=263$ ) or not (control group, $n=160$ ) during labor. There were no significant differences in age, BMI, history of previous vaginal delivery, gravidity, cervical Bishop score at admission, effacement $<=50$, oxytocin during labor, maternal comorbidities and prepartum laboratory test between the two groups. However, the factors including hospitals, time from last cesarean section, cervical dilation at admission $<3 \mathrm{~cm}$, and spontaneous onset of labor in study group were notably different from those in control group $(p<0.05)$ (see Table 1). Although these baseline factors were statistically different, subsequent univariate analysis, multivariate logistic regression and PSM may eliminate the effect of these confounding factors.

\section{Primary outcome of maternal delivery in TOLAC}

During the childbirth, there were two types of delivery according to multipara's status. As shown in Table 2, the VBAC rate was significantly higher in the study group than that in control group ( $85.55 \%$ vs. $69.38 \%, p<0.001)$. According to our coding, there was a higher rate of repeat cesarean sections in non-analgesia group than analgesia group $[(49 / 160,30.63 \%)$ vs. $(38 / 263,14.45 \%), p=$ 0.00153] due to fetal distress, stagnation of labor, fever or intrauterine infection, threatened uterine infection, unbearable pain, and change in fetal station.

\section{Secondary outcomes of maternal delivery in TOLAC}

For safety reasons, the hospital had established stringent non-labor induction standards and strictly controlled the use of oxytocin in TOLAC. So labor of multipara was mostly spontaneous. Each woman went through three stages during labor. From Table 3, the labor durations in study group were obviously lower than those in control group in the first and second stages of labor $(p<0.001)$, 
Table 2 Primary outcome of Maternal Delivery in TOLAC

\begin{tabular}{|c|c|c|c|c|c|}
\hline \multirow[t]{2}{*}{ Characteristics } & \multicolumn{2}{|c|}{ TOLAC (control) } & \multicolumn{2}{|c|}{ TOLAC (study) } & \multirow[t]{2}{*}{$P$ value } \\
\hline & $\begin{array}{l}\mathrm{N} \\
\text { (All) }\end{array}$ & $\mathrm{N}(\%)$ or Mean $\pm \mathrm{SD}$ & $\begin{array}{l}\mathrm{N} \\
\text { (All) }\end{array}$ & $\mathrm{N}(\%)$ or Mean $\pm \mathrm{SD}$ & \\
\hline Mode of delivery & 160 & & 263 & & $<0.001$ \\
\hline VBAC & & $111(69.38 \%)$ & & $225(85.55 \%)$ & \\
\hline Cesarean & & $49(30.63 \%)$ & & $38(14.45 \%)$ & \\
\hline Reason for cesarean & 49 & & 38 & & 0.00153 \\
\hline Fetal distress & & 10 & & 13 & \\
\hline Stagnation of labor & & 6 & & 7 & \\
\hline Fever or intrauterine infection & & 5 & & 13 & \\
\hline Threatened uterine rupture & & 3 & & 2 & \\
\hline Unbearable pain & & 23 & & 4 & \\
\hline Change in fetal station & & 2 & & 3 & \\
\hline
\end{tabular}

VBAC Vaginal birth after cesarean section

Data are mean \pm SD, or $\mathrm{n}(\%)$. SD Standard deviation

Comparisons were made using two-sided Student's t test or Wilcoxon rank sum test for non-normally distributed variables

Comparisons were made using Pearson's Chi-squared test and Fisher's exact test for proportions

Table 3 Secondary Outcomes of Maternal Delivery in TOLAC

\begin{tabular}{|c|c|c|c|c|c|c|}
\hline \multirow[t]{2}{*}{ Secondary outcomes } & \multicolumn{2}{|c|}{ TOLAC (control) } & \multicolumn{2}{|c|}{ TOLAC (study) } & \multirow[t]{2}{*}{ Statistics } & \multirow[t]{2}{*}{$P$ value } \\
\hline & $\begin{array}{l}\mathrm{N} \\
\text { (All) }\end{array}$ & $\mathrm{N}(\%)$ or Mean $\pm \mathrm{SD}$ & $\begin{array}{l}\mathrm{N} \\
\text { (All) }\end{array}$ & $\mathrm{N}(\%)$ or Mean $\pm \mathrm{SD}$ & & \\
\hline Initiating lactation period (h) & 157 & $11 \pm 13$ & 262 & $7 \pm 12$ & $24,276.5$ & 0.002 \\
\hline Uterine rupture & 160 & $0(0 \%)$ & 263 & $0(0 \%)$ & & 1 \\
\hline $\mathrm{PPH}$ & 154 & & 260 & & & 1 \\
\hline$<=500$ & & $151(98.05 \%)$ & & 255(98.08\%) & & \\
\hline$>500$ & & $3(1.95 \%)$ & & $5(1.92 \%)$ & & \\
\hline Episiotomy & 149 & $42(28.19 \%)$ & 256 & $111(43.36 \%)$ & & 0.003 \\
\hline Perineum/Cervical laceration & 149 & $70(46.98 \%)$ & 255 & $106(41.57 \%)$ & & 0.3 \\
\hline Instrumental delivery in VBAC & 27 & & 43 & & & 1 \\
\hline Vacuum extraction & & $15(13.51 \%)$ & & $23(10.22 \%)$ & & \\
\hline Forceps & & $12(10.81 \%)$ & & 20(8.89\%) & & \\
\hline \multicolumn{7}{|l|}{ Labor duration in VBAC } \\
\hline The first labor duration (min) & 108 & $334.14 \pm 225.94$ & 221 & $526.93 \pm 266.85$ & 6050.5 & $<0.001$ \\
\hline The second labor duration (min) & 102 & $28.09 \pm 31.62$ & 217 & $46.14 \pm 32.64$ & 5836 & $<0.001$ \\
\hline The third labor duration (min) & 108 & $8.62 \pm 3.87$ & 222 & $9.43 \pm 5.03$ & $11,211.5$ & 0.294 \\
\hline \multicolumn{7}{|l|}{ VAS pain at epidural } \\
\hline T0: time 0 & 99 & $6.34 \pm 2.16$ & 219 & $8.20 \pm 1.19$ & 5191.5 & $<0.001$ \\
\hline T1: PCEA after $30 \mathrm{~min}$ & 100 & $7.00 \pm 2.16$ & 217 & $0.94 \pm 1.61$ & 21,103 & $<0.001$ \\
\hline T2: cervical: $6 \mathrm{~cm}$ & 100 & $7.67 \pm 2.33$ & 211 & $1.09 \pm 1.71$ & $20,404.5$ & $<0.001$ \\
\hline T3: cervical: $10 \mathrm{~cm}$ & 99 & $8.64 \pm 2.13$ & 210 & $1.78 \pm 2.00$ & 20,213 & $<0.001$ \\
\hline T4: immediate delivery of the fetus & 97 & $1.65 \pm 2.44$ & 209 & $1.88 \pm 1.60$ & 8062 & 0.002 \\
\hline
\end{tabular}

PPH Postpartum hemorrhage, VAS Visual analog scale, VBAC Vaginal birth after cesarean section

Data are mean $\pm S D$, or $n(\%)$. SD Standard deviation

Comparisons were made using two-sided Student's t test or Wilcoxon rank sum test for non-normally distributed variables

Comparisons were made using Pearson's Chi-squared test and Fisher's exact test for proportions 
while the labor duration between the two group in the third stage of labor was similar $(p=0.294)$. Instrumentassisted delivery could be used when delivery was too long. In women who had a successful VBAC, the rate of instrumental delivery was $16.5 \%$ (70/423), with $16.8 \%$ $(27 / 160)$ in control group and $16.3 \%(43 / 263)$ in study group, indicating no significant difference $(p=1)$. There were no differences in uterine rupture, $\mathrm{PPH}$, perineum or cervical laceration between the two groups. In addition, the occurrence of episiotomy was higher for multiparas with epidural than that without epidural $(43.36 \%$ vs. $28.19 \%, p<0.05)$, and the initiating lactation period was delayed in control group $(7 \pm 12 \mathrm{~h}$ vs. $11 \pm 13$ $\mathrm{h}, p<0.001)$. At the beginning, the mean VAS score of epidural pain in control group was notably lower than that in study group $(6.34 \pm 2.16$ vs. $8.20 \pm 1.19, p<$ 0.001 ). Thirty minutes later, the mean VAS score for epidural analgesia group fell from $8.20 \pm 1.19$ to $0.94 \pm$ 1.61 , while for non-epidural analgesia group, the mean VAS score increased from $6.34 \pm 2.16$ to $7.00 \pm 2.16$. When the uterus expanded to $6 \mathrm{~cm}$ and $10 \mathrm{~cm}$, the mean VAS pain scores in study group were significantly lower than those in control group $[(1.09 \pm 1.71)$ vs. $(7.67 \pm$ $2.33)$; $(1.78 \pm 2.00)$ vs. $(8.64 \pm 2.13), p<0.001]$. However, when the immediate delivery of fetus, the mean VAS pain scores were reversed in the two groups $(p<0.002)$.

\section{Neonatal outcomes in TOLAC}

Neonates in both groups were of similar weight and Apgar scores. The limitation of Apgar score may cause misdiagnosis and missed diagnosis of neonatal asphyxia.
To make up for the deficiency, umbilical arterial blood $\mathrm{pH}$ value was also adopted in the diagnosis of neonatal asphyxia [20]. As shown in Table 4, the number of neonates whose $\mathrm{pH}$ value was lower than 7.2 in control group was remarkably higher than that in study group, while the number of neonates with $\mathrm{pH}>7.2$ was reversed $(p=0.001)$. The normal PCO2 score is between 35 and 45 . It was obvious that the score in control group was above normal, which was significantly higher than that in study group $(46.50 \pm 10.54$ vs. $43.80 \pm 10.44, p=$ 0.041 ). In addition, the base excess required by the study group was significant less than the control group $(p<$ 0.001). These results suggested that the physical conditions of neonates born to multipara who received epidural analgesia were better.

\section{Univariate and multivariate analysis of mode of delivery in TOLAC}

Mode of delivery of multiparas in TOLAC was the dependent variable, epidural analgesia and other confounding factors were the independent variables. The univariate analysis in Table 5 showed that epidural analgesia, hospital, age, cervical dilation, cervical score at admission, effacement, the onset of labor, progress of labor in previous caesarean section (cervical dilation: $<3 \mathrm{~cm}$ ) and neonatal weight were associated with mode of delivery in TOLAC. However, it was not clear whether these indices play an independent role in the mode of delivery or act in combination with other factors. Thus, we analyzed the above indices with multivariate logistic regression. The results revealed that the correction of

Table 4 Neonatal Outcomes in TOLAC

\begin{tabular}{|c|c|c|c|c|c|c|}
\hline \multirow[t]{2}{*}{ Characteristic } & \multicolumn{2}{|c|}{ TOLAC (control) } & \multicolumn{2}{|c|}{ TOLAC (study) } & \multirow[t]{2}{*}{ Statistics } & \multirow[t]{2}{*}{$P$ value } \\
\hline & $\begin{array}{l}\mathrm{N} \\
\text { (All) }\end{array}$ & $\mathrm{N}(\%)$ or Mean $\pm \mathrm{SD}$ & $\begin{array}{l}N \\
\text { (All) }\end{array}$ & $\mathrm{N}(\%)$ or Mean $\pm \mathrm{SD}$ & & \\
\hline \multicolumn{7}{|l|}{ Neonatal results } \\
\hline Weight & 148 & & 250 & & & 0.903 \\
\hline$<3500$ & & $114(77.03 \%)$ & & 190(76.00\%) & & \\
\hline$\geq 3500$ & & $34(22.97 \%)$ & & $60(24.00 \%)$ & & \\
\hline Admission to neonatal ward after birth & 153 & $12(7.84 \%)$ & 256 & $20(7.81 \%)$ & & 1 \\
\hline 1 min Apgar & 159 & $0(I R Q)$ & 261 & $0(I R Q)$ & $12,485.5$ & 0.535 \\
\hline 5 min Apgar & 159 & $0(I R Q)$ & 261 & $0(I R Q)$ & 12,542 & 0.292 \\
\hline \multicolumn{7}{|l|}{ Umbilical arterial blood } \\
\hline $\mathrm{PH}$ & 119 & & 220 & & & 0.001 \\
\hline$<7.2$ & & $20(16.81 \%)$ & & $12(5.45 \%)$ & & \\
\hline$>=7.2$ & & $99(83.19 \%)$ & & 208(94.55\%) & & \\
\hline PCO2 $(\mathrm{mmHg})$ & 118 & $46.50 \pm 10.54$ & 220 & $43.80 \pm 10.44$ & 14,729 & 0.041 \\
\hline Base excess (BE. mmol. L) & 120 & $-6.04 \pm 3.06$ & 220 & $-4.38 \pm 3.00$ & 8989 & $<0.001$ \\
\hline
\end{tabular}

$I Q R$ Interquartile range

Data are Mean \pm SD, or $n(\%)$

Comparisons were made using two-sided Student's t test or Wilcoxon rank sum test for non-normally distributed variables

Comparisons were made using Pearson's Chi-squared test and Fisher's exact test for proportions 
Table 5 Univariate and Multivariate analysis of Mode of delivery in TOLAC

\begin{tabular}{|c|c|c|c|c|}
\hline \multirow[t]{2}{*}{ Variable } & \multicolumn{2}{|c|}{ Univariate } & \multicolumn{2}{|c|}{ Multivariate $(n=354)$} \\
\hline & $P$ & $\mathrm{OR}(95 \% \mathrm{Cl})$ & $P$ & $\mathrm{OR}(95 \% \mathrm{Cl})$ \\
\hline Epidural analgesia & $<0.001$ & $2.614(1.620-4.248)$ & 0.006 & $2.590(1.315-5.165)$ \\
\hline \multicolumn{5}{|l|}{ Hospital name } \\
\hline $\mathrm{MCH}$ & & 1 (reference) & & 1 (reference) \\
\hline BA & 0.473 & 1.340(0.623-3.197) & 0.141 & 2.253(0.799-7.068) \\
\hline LG & $<0.001$ & $0.214(0.115-0.396)$ & $<0.001$ & $0.138(0.046-0.391)$ \\
\hline \multicolumn{5}{|l|}{ General information } \\
\hline Age $(y)$ & 0.015 & $0.921(0.861-0.983)$ & $<0.001$ & $0.853(0.774-0.933)$ \\
\hline Gestational age (day) & 0.421 & $0.990(0.965-1.011)$ & & \\
\hline $\mathrm{BMI}\left(\mathrm{kg} / \mathrm{m}^{2}\right)$ & 0.129 & $0.944(0.875-1.018)$ & & \\
\hline Maternal education $>12 \mathrm{y}$ & 0.187 & $0.681(0.375-1.184)$ & & \\
\hline Husband education $>12$ y & 0.114 & 0.604(0.312-1.099) & & \\
\hline Housewives & 0.193 & $2.036(0.776-7.000)$ & & \\
\hline \multicolumn{5}{|l|}{ History of pregnancy and childbirth } \\
\hline History of previous vaginal delivery & 0.615 & $0.796(0.343-2.076)$ & & \\
\hline \multicolumn{5}{|l|}{ Gravidity time (times) } \\
\hline 2 & & 1 (reference) & & \\
\hline 3 & 0.78 & 1.076(0.641-1.804) & & \\
\hline$>3$ & 0.971 & $1.013(0.514-2.083)$ & & \\
\hline \multicolumn{5}{|l|}{ Time from last cesarean section (year) } \\
\hline$\leq 3$ & & 1 (reference) & & \\
\hline $3-5$ & 0.246 & $2.282(0.477-8.592)$ & & \\
\hline$>5$ & 0.923 & $1.071(0.223-4.046)$ & & \\
\hline \multicolumn{5}{|c|}{ Progress of Labor in previous caesarean section (cervical dilation: $\mathrm{cm}$ ) } \\
\hline 0 & & 1 (reference) & & \\
\hline$<3$ & 0.029 & $1.882(1.083-3.381)$ & & \\
\hline$\geq 3$ & 0.148 & $1.827(0.844-4.417)$ & & \\
\hline 10 & 0.062 & $6.942(1.391-126.155)$ & & \\
\hline Cervical score at admission (score) & 0.003 & $1.203(1.066-1.360)$ & $<0.001$ & $1.421(1.177-1.727)$ \\
\hline Cervical dilation at admission $(\mathrm{cm}) \geq 3$ & 0.021 & $2.629(1.232-6.513)$ & 0.058 & $2.698(1.026-8.200)$ \\
\hline Effacement & 0.027 & $1.750(1.062-2.865)$ & & \\
\hline The onset of labor: Spontaneous & $<0.001$ & $7.428(3.484-16.431)$ & 0.002 & $6.801(2.093-23.518)$ \\
\hline Oxytocin during labor & 0.044 & $0.451(0.211-1.011)$ & & \\
\hline HGB (g/L:115-150) & 0.843 & 0.998(0.981-1.018) & & \\
\hline PLT (125-350) & 0.924 & $1.000(0.996-1.005)$ & & \\
\hline \multicolumn{5}{|l|}{ Labor duration } \\
\hline The first labor duration (min) & 0.553 & 1.003(0.996-1.018) & & \\
\hline The second labor duration (min) & 0.37 & $1.157(0.983-1.945)$ & & \\
\hline The third labor duration (min) & 0.859 & 0.970(0.771-1.779) & & \\
\hline \multicolumn{5}{|l|}{ Neonatal } \\
\hline Weight $(\mathrm{g}) \geq 3500$ & 0.015 & $0.512(0.301-0.884)$ & 0.003 & $0.360(0.183-0.711)$ \\
\hline
\end{tabular}

OR Odds ratio, Cl Confidence interval;

Multivariable logistic regression included entered factors variable as $p<0.05$ (Epidural analgesia, Hospital, Age, Progress of Labor in previous caesarean section, Cervical score at admission, cervical dilation at admission, effacement, the onset of labor: Spontaneous, Oxytocin during labor, Neonatal Weight) and using stepwise regression by AIC criteria. Hosmer-Lemeshow goodness of fit (GOF) test: Chi-squared $=8.034, \mathrm{df}=8, P$ value $=0.430 \mathrm{McFadden}$ 's pseudo-R squared $=$ 0.249 Cox \& Snell pseudo-R squared $=0.223$ Nagelkerke pseudo-R squared $=0.350$. All VIFs $<2$ 
confounding factors including epidural analgesia, hospital, age, cervical Bishop score at admission, spontaneous onset of labor, and neonatal weight were still shown as promotion probability in epidural analgesia group $(\mathrm{OR}=2.590,0.138,0.853,1.421,6.801$ and 0.360 , respectively; $95 \% \mathrm{CI}=1.315-5.165,0.046-0.391,0.774-$ $0.933,1.177-1.727,2.093-23.518$ and $0.183-0.711$, respectively; $p=0.006, p<0.001, p<0.001, p<0.001, p=$ 0.002 and $p=0.003$, respectively). The multivariate logistic regression model was proved well fitness by Hosmer and Lemeshow goodness of fit (GOF) test $(p=0.43)$, and the expansion factor of each factor was $\operatorname{VIF}<2$.

\section{Univariate and multivariate analysis of mode of delivery in TOLAC matched by PSM}

Furthermore, PSM was used to reduce the potential selection bias and validate the results again. We used 1:1 nearest neighbor matching method to select 118 multiparas in study group (96 unmatched) and 118 multiparas with confounding factors in control group (see Table 6).

After matching, the univariate analysis showed that epidural analgesia was still the promotion factor of spontaneous labor $[\mathrm{OR}=4.361(2.202-9.198), p<0.001]$. In addition, multivariate logistic regression model after the stepwise regression revealed that the correction of confounding factors including epidural analgesia, cervical Bishop score at admission and spontaneous onset of labor were still shown as promotion probability in study group $(\mathrm{OR}=4.480,1.360$, and 10.188 , respectively; $95 \% \mathrm{CI}=2.025-10.660,1.113-1.673$, and $2.875-48.418$, respectively; $p<0.001, p=0.003$, and $p<0.001$, respectively) (Fig. 2). The multivariate logistic regression model was proved well fitness by Hosmer-Lemeshow goodness of fit (GOF) test $(p=0.092)$, and the expansion factor of each factor was $\mathrm{VIF}<2$.

As shown in Fig. 3, the two multivariate logistic regression was performed to predict the VBAC in TOLAC. The model without PSM showed that the area under the curve (AUC) was $0.822(95 \% \mathrm{CI}=0.763-0.882)$ with sensitivity of 0.833 , specificity of 0.694 , positive predictive value (PPV) of 0.914 , and negative predictive value (NPV) of 0.515. The model with PSM showed that AUC was $0.816(95 \% \mathrm{CI}=0.7481-0.8841)$ with sensitivity of 0.702 , specificity of 0.804 , PPV of 0.929 , and NPV of 0.427 .

\section{Discussion}

Labor is a painful experience, and about $30 \%$ of mothers have found it more painful than expected [21]. The previous cesarean section increases maternal emotional stress and affect their initial consents to TOLAC, while subsequent VBAC increases maternal satisfaction and motivation for future vaginal delivery [22]. In this study, we found that epidural analgesia remarkably increased the success rate of VBAC after TOLAC, and revealed some critical protective factors including cervical Bishop score at admission, spontaneous onset of labor, which showed that probability of VBAC with epidural analgesia was 2.027 times as non-epidural after TOLAC. Oxytocin was used in patients with weak contractions during labor, and no uterine rupture was found. However, most cases of vaginal trial were mainly normal labor. Lower acceptance and lack of experience of TOLAC in the mainland of China result in strict inclusion criteria for TOLAC eligibility might be the reason. This study also found that cervical conditions during delivery (Bishop score) had a positive correlation with vaginal delivery success rate. Consistently, Smith GCS et al. have clarified that parturients whose cervix is more than $4 \mathrm{~cm}$ and the cervical canal opens more than $25 \%$ in TOLAC received higher success rate [23].

The effect of multiparas' age on TOLAC has been analyzed in many studies, but no uniform results. The study conducted by Regan, J et al. [24] have revealed that maternal age is not associated with the success or failure of VBAC in low-risk women. However, Minsart et al. [25] have proposed that when multivariate models were included, the independent factor associated with TOLAC was maternal age $<35$ years. Moreover, Sentilhes et al. [8] have indicated that TOLAC's failure rate increases as pregnant women age. Smith have also reported that with a 5-year old increase, the OR was adjusted of vaginal trial failure $1.22,95 \%$ CI: $1.16-1.28$, which was in line with our research [23].

Breast milk contains essential nutrients for the growth and development of infants. However, the pain during labor may delay lactogenesis [26]. Thus, initiating lactation period was a necessary index evaluating the effect of epidural analgesia. In the present study, the initiating lactation period was shorter in epidural group than nonepidural group. It was demonstrated that early or late initiation of epidural analgesia for labor had similar effects on all measured outcomes, which was inconsistent with our results regarding the initiating lactation period [27-29]. Previous study has indicated that the timely and effective analgesia after delivery helps the multipara to take the comfortable position so that newborn could suck nipple frequently, which was conducive to milk secretion [30].

Episiotomy is not supposed to be performed routinely, whereas it is inevitably required in many cases of operative vaginal delivery [31]. It is reported that episiotomy occurs in $65.8 \%$ of cases undergoing operative vaginal delivery [32]. In the present study, episiotomy usage rate was $43.36 \%$ in epidural group which was higher than $28.19 \%$ in non-epidural group, and the mild prolongation of labor did not increase the adverse effects of maternal and neonatal, no uterine rupture occurred as well. 
Table 6 Univariate and Multivariate analysis of Mode of delivery in TOLAC matched by PSM

\begin{tabular}{|c|c|c|c|c|}
\hline \multirow[t]{2}{*}{ Variable } & \multicolumn{2}{|c|}{ Univariate $(n=236)$} & \multicolumn{2}{|c|}{ Multivariate $(n=236)$} \\
\hline & $P$ & $\mathrm{OR}(95 \% \mathrm{Cl})$ & $P$ & OR $(95 \% \mathrm{Cl})$ \\
\hline Epidural analgesia & $<0.001$ & $4.361(2.202-9.198)$ & $<0.001$ & $4.480(2.025-10.660)$ \\
\hline \multicolumn{5}{|l|}{ Hospital name } \\
\hline $\mathrm{MCH}$ & & 1 (reference) & & \\
\hline BA & 0.608 & $1.265(0.539-3.331)$ & & \\
\hline LG & 0.984 & $0.000(N A-13382569087647956.000)$ & & \\
\hline \multicolumn{5}{|l|}{ General information } \\
\hline Age $(y)$ & 0.892 & 1.006(0.923-1.095) & & \\
\hline Gestational age (day) & 0.443 & $0.987(0.952-1.014)$ & & \\
\hline $\mathrm{BMI}\left(\mathrm{kg} / \mathrm{m}^{2}\right)$ & 0.211 & $0.940(0.851-1.038)$ & & \\
\hline Maternal education $>12 \mathrm{y}$ & 0.078 & $0.494(0.213-1.042)$ & & \\
\hline Husband education $>12$ y & 0.283 & $0.648(0.278-1.380)$ & & \\
\hline Housewives & 0.523 & $1.515(0.480-6.705)$ & & \\
\hline \multicolumn{5}{|l|}{ History of pregnancy and childbirth } \\
\hline History of previous vaginal delivery & 0.203 & $2.641(0.728-16.976)$ & & \\
\hline \multicolumn{5}{|l|}{ Gravidity time (times) } \\
\hline 2 & & 1 (reference) & & \\
\hline 3 & 0.462 & $0.776(0.389-1.511)$ & & \\
\hline$>3$ & 0.237 & $2.027(0.682-7.493)$ & & \\
\hline \multicolumn{5}{|l|}{ Time from last cesarean section (year) } \\
\hline$\leq 3$ & & 1 (reference) & & \\
\hline $3-5$ & 0.188 & $2.723(0.532-11.823)$ & & \\
\hline$>5$ & 0.496 & $1.691(0.325-7.484)$ & & \\
\hline \multicolumn{5}{|c|}{ Progress of Labor in previous caesarean section (cervical dilation: $\mathrm{cm}$ ) } \\
\hline 0 & & 1 (reference) & & 1 (reference) \\
\hline$<3$ & 0.013 & $2.601(1.260-5.731)$ & 0.168 & $1.811(0.794-4.361)$ \\
\hline$\geq 3$ & 0.462 & $1.451(0.565-4.235)$ & 0.892 & $0.927(0.320-2.974)$ \\
\hline 10 & 0.988 & $17,640,531.947(0.000-N A)$ & 0.988 & $9,284,640.473(0.000-N A)$ \\
\hline Cervical score at admission (score) & 0.004 & $1.269(1.082-1.494)$ & 0.003 & $1.360(1.113-1.673)$ \\
\hline Cervical dilation at admission $(\mathrm{cm}) \geq 3$ & 0.068 & $2.742(1.028-9.522)$ & & \\
\hline Effacement & 0.927 & 1.034(0.493-2.069) & & \\
\hline The onset of labor: Spontaneous & $<0.001$ & 20.754(6.329-93.768) & $<0.001$ & $10.188(2.875-48.418)$ \\
\hline Oxytocin during labor & 0.487 & 0.719(0.295-1.939) & & \\
\hline HGB (g/L:115-150) & 0.592 & 0.994(0.973-1.017) & & \\
\hline PLT (125-350) & 0.62 & 0.999(0.993-1.004) & & \\
\hline \multicolumn{5}{|l|}{ Neonatal } \\
\hline Weight(g) $\geq 3500$ & 0.304 & $0.682(0.334-1.452)$ & & \\
\hline
\end{tabular}

OR Odds ratio, $\mathrm{Cl}$ Confidence interval, PSM Propensity score matching

Data had been matched by using propensity score matching with 1:1 nearest neighbor matching. Multivariable logistic regression after PSM included entered factors variable as $p<0.05$ (Epidural analgesia, Progress of Labor in previous caesarean section, Cervical score at admission, the onset of labor: Spontaneous) and using stepwise regression by AIC criteria. Hosmer-Lemeshow goodness of fit (GOF) test: Chi-squared $=13.627, \mathrm{df}=8, P$ value $=0.092 \mathrm{McF}$ adden's pseudo-R squared $=0.232$ Cox \& Snell pseudo-R squared $=0.215$ Nagelkerke pseudo-R squared $=0.332$. All VIFs $<2$

It is well known that reduction in uteroplacental perfusion conferred by uterine contractions during labor can increase the risk of asphyxia, neurological injury and death of fetuses. Moreover, long duration of labor may increase the risk of uterine rupture. Hence, long duration of labor should be avoided during VBAC through shortening the second stage of labor by operative vaginal delivery, further reducing the incidence of fetal distress 


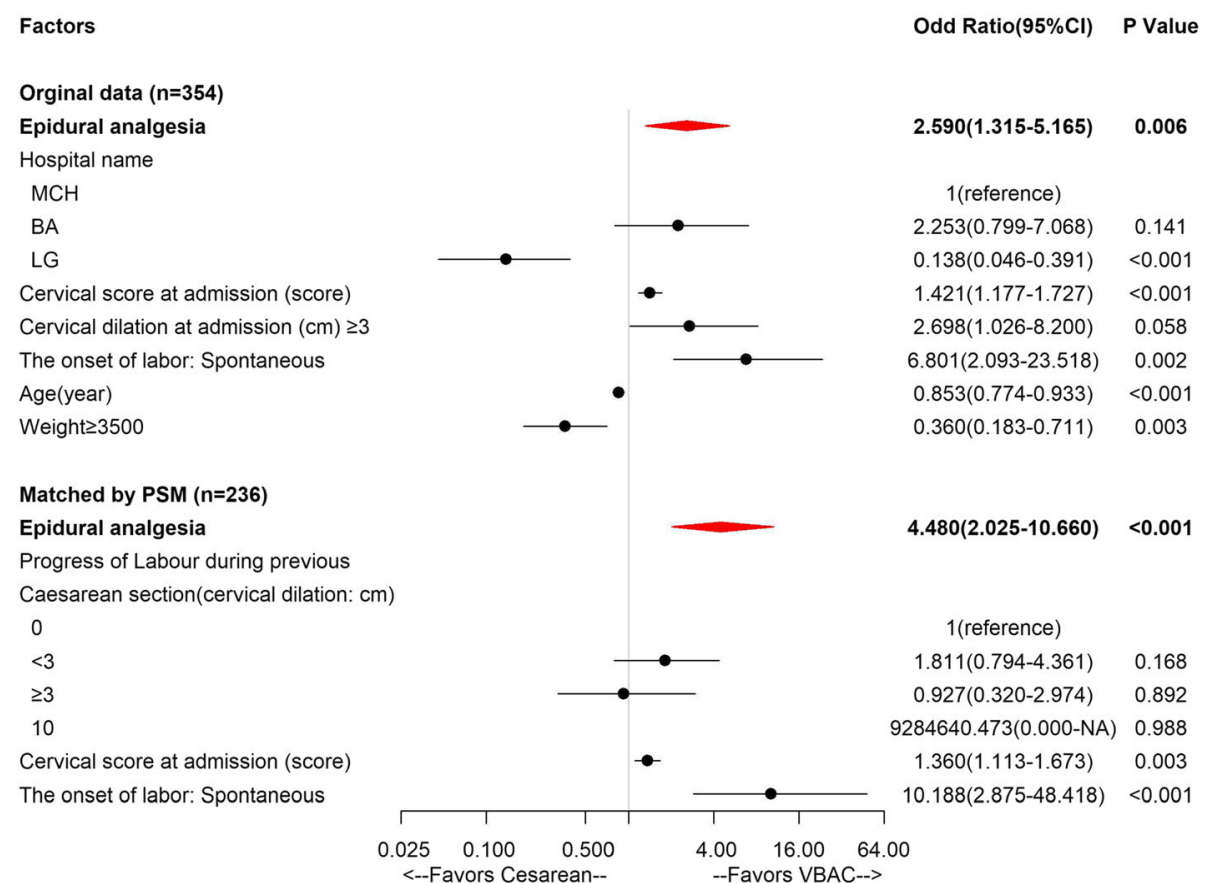

Fig. 2 ROC of univariate and multivariate analysis of mode of delivery in TOLAC matched by PSM

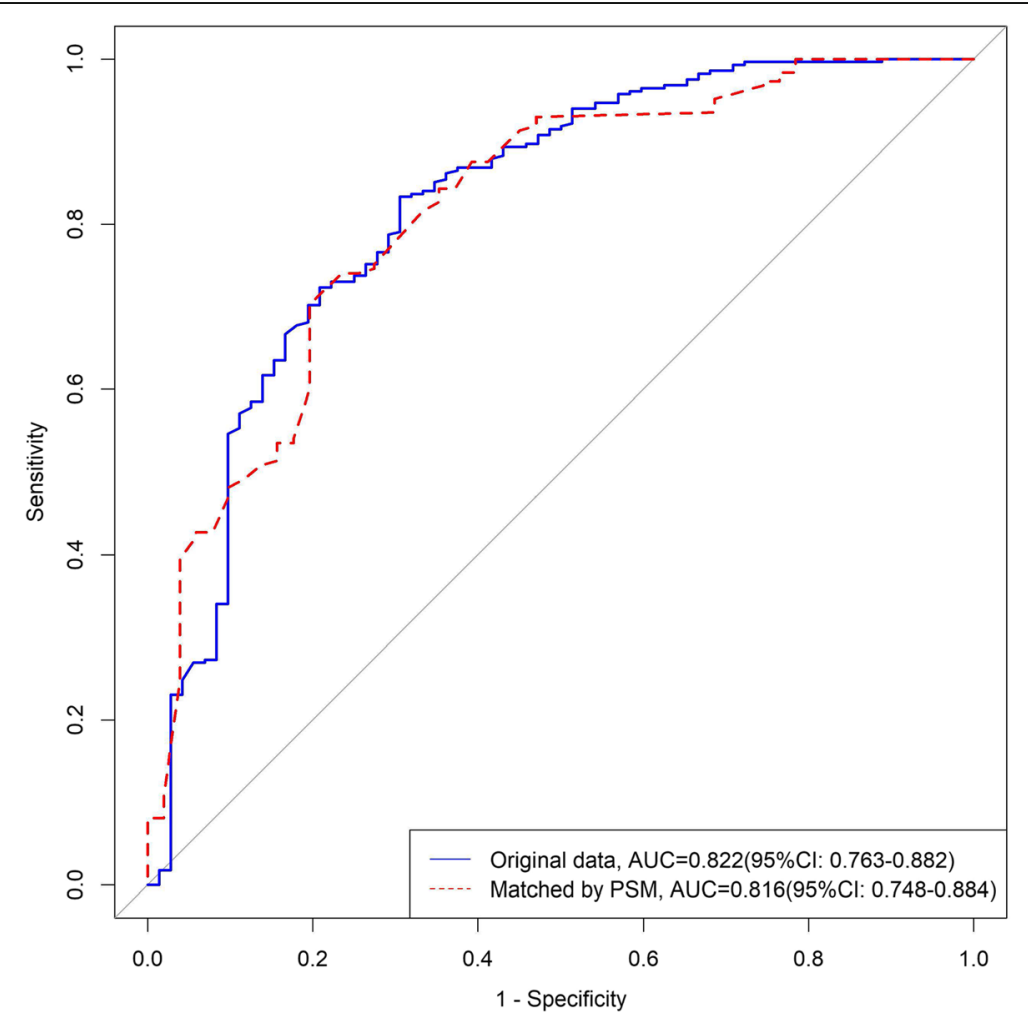

Fig. 3 Two multivariate logistic regression was performed to predict the VBAC in TOLAC 
[33]. Notably, a natural experiment conducted by J. Zhang et al. [34] has demonstrated that the second stage of labor is significantly longer by about $25 \mathrm{~min}$, which is consistent with this study. The reason may be that during the second stage of labor, in addition to uterine contraction, pelvic floor muscles, active breath holding and other functions of multiparas are also needed, while epidural analgesia weakens the function of pelvic floor muscles and increases abnormal fetal position after delivery [35].

The overall epidural analgesia rate in this study was 62.17\%. Among them, the Affiliated Shenzhen Maternity \& Child Healthcare Hospital was 57.74\%, the Bao'an Maternal and Child Health Hospital was 98\%, and the Longgang District Maternity \& Child Healthcare Hospital of Shenzhen City was $47.17 \%$. The high rate of epidural analgesia in Bao'an Maternal and Child Health Hospital is mainly due to its comprehensive promotion of "painless hospital" construction, which is in response to the call of the world health organization "improve the painless delivery rate". As long as there is no contraindication for intravertebral labor analgesia, all parturients could perform painless delivery. If a parturient refuse to give birth painlessly, she will have to go to another hospital, which reflects the differences between culture in Eastern and Western.

There are several limitations in this study. First, the multi-center study enriched the sample size, but different labor modes and labor analgesia management might influence the incidence of epidural analgesia and the high incidence of episiotomy. Notably, some midwives performed an active lateral incision to prevent tearing of the vulva. Therefore, there were some subjective factors in the implementation of lateral incision. Further experimental design should fully plan the surgery process, so that all operations are strictly unified. Second, the analyzed multiparas in this study were of the same ethnicity. There was a significant difference in the proportion of patients with painless labor in the three hospitals, indicating that the three hospitals had some selection bias in the patients with painless labor. In the follow-up process, multivariate logistic regression and PSM were performed to correct the impact of inter-hospital and other confounding factors. However, due to the sample size, the problem of selection bias of the previous sample itself cannot be completely eliminated (the coverage area of the hospital was different). Hence, the sample size needs to be further increased in the future to further verify this result. Third, observational cohort studies do not yield causality and can only see correlation analysis.

\section{Conclusions}

Epidural analgesia could reduce labor pain, and no increased risk of postpartum bleeding or uterine rupture, as well as adverse effects in newborns were observed. The labor duration of multiparas was increased, but within acceptable range. In summary, epidural analgesia may be safe for both mother and neonate in the three studied hospitals. The data of present study can inform clinical delivery practice. However, in their provision of care medical practitioners should specifically assess the desires and expectations of the laboring women.

\section{Abbreviations \\ ACOG: American Association of Obstetricians and Gynecologists; AUC: Area under the curve; FHRs: Abnormal fetal heart rates; GOF: Goodness of fit; NPV: Negative predictive value; PPH: Postpartum hemorrhage; PPV: Positive predictive value; PSM: Propensity score matching; RCS: Repeated cesarean section; TOLAC: The trial of labor after cesarean section; VAS: Visual Analogue Score; VBAC: Vaginal birth after cesarean}

\section{Acknowledgements}

We thank Yu Lin (Statistician, Shenzhen Withsum Technology Limited, supported by Shenzhen Science and Technology Plan, Grant No. CKCY20180323174659823) for assistance with data analysis that greatly improved the manuscript.

\section{Authors' contributions}

JS participated in the design and conduct of the experiment, editing the manuscript, reviewing and approving the final manuscript. XY organized and analyzed the data, wrote, reviewed and approved the final manuscript. AY helped design and conduct the study, write and approve the final manuscript. YL helped design the study write manuscript, review and approve the final manuscript. YX helped conduct the study, write the manuscript, review and approve the final manuscript. LZ helped organize and analyze the data, write and approve the final manuscript. DL helped collect and analyze the data, write and approve the final manuscript. TH helped analyze the data, write and approve the final manuscript. $\mathrm{ZZ}$ and $\mathrm{XH}$ helped organize the data, write and approve the final manuscript. All authors have read and approved the manuscript.

\section{Funding}

No funding was obtained for this study.

\section{Availability of data and materials}

The datasets compiled during the current study are available from the corresponding author on reasonable request.

\section{Ethics approval and consent to participate}

The study was approved by the human research committee of Affiliated Shenzhen Maternity \& Child Healthcare Hospital, Bao'an Maternal and Child Health Hospital, and Longgang District Maternity \& Child Healthcare Hospital of Shenzhen City. Written informed consent was obtained from all individual participants included in the study.

\section{Consent for publication}

Not applicable.

\section{Competing interests}

The authors declare that they no competing interests.

\section{Author details}

${ }^{1}$ Department of Anesthesiology, Affiliated Shenzhen Maternity \& Child Healthcare Hospital, Southern Medical University, No.2004 Hongli Road, Futian District, Shenzhen 518028, Guangdong, China. ${ }^{2}$ Department of Anesthesiology, Bao'an Maternal and Child Health Hospital, Jinan University, Shenzhen 518100, China. ${ }^{3}$ Department of Anesthesiology, Longgang District Maternity \& Child Healthcare Hospital of Shenzhen City, Shenzhen 518172 , China. ${ }^{4}$ Department of Obstetrics, Affiliated Shenzhen Maternity \& Child Healthcare Hospital, Southern Medical University, Shenzhen 518028, Guangdong, China. 
Received: 19 August 2019 Accepted: 28 November 2019

Published online: 16 December 2019

\section{References}

1. Project E. European perinatal health report: the health and care of pregnant women and babies in Europe (2010). EURO-PERISTAT Project.

2. Quinlan JD, Murphy NJ. Cesarean delivery: counseling issues and complication management. Am Fam Physician. 2015;91(3):178-84.

3. Ye J, Betrán AP, Guerrero Vela M, et al. Searching for the Optimal Rate of Medically Necessary Cesarean Delivery[J]. Birth. 2014;41(3):237-244.

4 Fawsitt CG, Bourke J, Greene RA, Everard CM, Murphy A, Lutomski JE. A what price? A cost-effectiveness analysis comparing trial of labour after previous caesarean versus elective repeat caesarean delivery. PLoS One. 2013;8(3):e58577

5. Costantine MM, Karin F, Byers BD, et al. Validation of the prediction model for success of vaginal birth after cesarean delivery. Obstet Gynecol. 2009; 114(5):1029-33.

6. Grobman WA, Yinglei L, Landon MB, et al. Does information available at admission for delivery improve prediction of vaginal birth after cesarean? Am J Perinatol. 2009:26(10):693-701.

7. Jing W, Otten $H$, Sullivan L, Lovellsimons L, Granekcatarivas M, Fritzsche K. Improving the doctor-patient relationship in China: the role of Balint groups. Int J Psychiatry Med. 2013;46(4):417-27.

8. Sentilhes L, Vayssière C, Beucher $\mathrm{G}$, et al. Delivery for women with a previous cesarean: guidelines for clinical practice from the French College of Gynecologists and Obstetricians (CNGOF). Eur J Obstet Gynecol Reprod Biol. 2013;170(1):25-32

9. Goetzl LM. ACOG practice bulletin. Clinical management guidelines for obstetrician-gynecologists number 36, July 2002. Obstetric analgesia and anesthesia. Obstet Gynecol. 2002;100(1):177-91.

10. Alexander JM, Sharma SK, McIntire DD, Wiley J, Leveno KJ. Intensity of labor pain and cesarean delivery. Anesth Analg. 2001;92(6):1524-8.

11. Panni MK, Segal S. Local anesthetic requirements are greater in dystocia than in normal labor. Anesthesiology. 2003;98(4):957-63.

12. Grant EN, Tao W, Craig M, McIntire D, Leveno K. Neuraxial analgesia effects on labour progression: facts, fallacies, uncertainties and the future. BJOG. 2015;122(3):288-93.

13. Lederman RP, Lederman E, Work BA, Mccann DS. Plasma epinephrine, norepinephrine, and cortisol of multigravidas in pregnancy and labor and in umbilical cord arteries after delivery. J Psychosom Obstet Gynecol. 2009; 10(1):57-69.

14. Lederman RP, Lederman E, Work BA Jr, McCann DS. The relationship of maternal anxiety, plasma catecholamines, and plasma cortisol to progress in labor. Am J Obstet Gynecol. 1978;132(5):495-500

15. Neumark J, Hammerle AF, Biegelmayer C. Effects of epidural analgesia on plasma catecholamines and cortisol in parturition. Acta Anaesthesiol Scand. 2010;29(6):555-9.

16. Jouppila R, Hollmen A. The effect of segmental epidural analgesia on maternal and foetal acid-base balance, lactate, serum potassium and creatine phosphokinase during labour. Acta Anaesthesiol Scand. 1976;20(3):259-68

17. Jones L. Pain management for women in labour: an overview of systematic reviews[J]. Cochrane Database of Systematic Reviews. 2012;5(2):101-102.

18. Konefał H, Jaskot B, Czeszyńska MB, Pastuszka J. Remifentanil patientcontrolled analgesia for labor - monitoring of newborn heart rate, blood pressure and oxygen saturation during the first 24 hours after delivery. Arch Med Sci. 2013;9(4):697-702.

19. Tintara $H$, Voradithi $P$, Choobun T. Effectiveness of celecoxib for pain relief and antipyresis in second trimester medical abortions with misoprostol: a randomized controlled trial. Arch Gynecol Obstet. 2018;297(3):709-15.

20. Yeh P, Emary K, Impey L. The relationship between umbilical cord arterial $\mathrm{pH}$ and serious adverse neonatal outcome: analysis of 51,519 consecutive validated samples. BJOG. 2012;119(7):824-31.

21. Dunn EA, O'Herlihy C. Comparison of maternal satisfaction following vaginal delivery after caesarean section and caesarean section after previous vaginal delivery. Eur J Obstet Gynecol Reprod Biol. 2005;121(1):56-60.

22. Hung TH, Hsieh TT, Liu HP. Differential effects of epidural analgesia on modes of delivery and perinatal outcomes between nulliparous and multiparous women: a retrospective cohort study. PLoS One. 2015;10(3): e0120907.
23. Smith GCS, White IR, Pell JP, Dobbie R. Predicting cesarean section and uterine rupture among women attempting vaginal birth after prior cesarean section. PLoS Med. 2005;2(9):e252.

24. Regan J, Wolfe K, Snyder C, Thompson A, Defranco E. 659: VBAC success in low risk women. Am J Obstet Gynecol. 2012;206(1):S295.

25. Minsart AF, Liu H, Moffett S, Chen C, Ji N. Vaginal birth after caesarean delivery in Chinese women and Western immigrants in Shanghai. J Obstet Gynaecol. 2017;37(4):446-9.

26. Evans KC, Evans RG, Royal R, Esterman AJ, James SL. Effect of caesarean section on breast milk transfer to the normal term newborn over the first week of life. Arch Dis Child Fetal Neonatal Ed. 2003:88(5):F380-2.

27. Wassen MM, Zuijlen J, Roumen FJ, Smits LJ, Marcus MA, Nijhuis JG. Early versus late epidural analgesia and risk of instrumental delivery in nulliparous women: a systematic review. BJOG. 2011;118(6):655-61.

28. Wang F, Shen X, Guo X, Peng Y, Gu X. Labor analgesia examining G. epidural analgesia in the latent phase of labor and the risk of cesarean delivery: a five-year randomized controlled trial. Anesthesiology. 2009;111(4):871-80.

29. Sng BL, Leong $W L$, Zeng $Y$, et al. Early versus late initiation of epidural analgesia for labour. Cochrane Database Syst Rev. 2014;10:CD007238.

30. Mizuno K, Fujimaki K, Sawada M. Sucking behavior at breast during the early newborn period affects later breast-feeding rate and duration of breast-feeding. Pediatr Int. 2004;46(1):15-20.

31. Committee on Practice B-O. ACOG Practice Bulletin No. 154: operative vaginal delivery. Obstet Gynecol. 2015;126(5):e56-65.

32. Shmueli A, Benziv RG, Hiersch L, et al. Episiotomy - risk factors and outcomes. J Matern Fetal Neonatal Med. 2017;30(3):251-6.

33. Shmueli A, Gabbay Benziv R, Hiersch L, et al. Episiotomy - risk factors and outcomes(). J Matern Fetal Neonatal Med. 2017;30(3):251-6.

34. Zhang J, Yancey MK, Klebanoff MA, Schwarz J, Schweitzer D. Does epidural analgesia prolong labor and increase risk of cesarean delivery? A natura experiment. Am J Obstet Gynecol. 2001;185(1):128-34.

35. Zhang G, Feng Y. Effect of epidural analgesia on the duration of labor stages and delivery outcome. J Southern Med Univ. 2012;32(8):1218-20.

\section{Publisher's Note}

Springer Nature remains neutral with regard to jurisdictional claims in published maps and institutional affiliations.

Ready to submit your research? Choose BMC and benefit from:

- fast, convenient online submission

- thorough peer review by experienced researchers in your field

- rapid publication on acceptance

- support for research data, including large and complex data types

- gold Open Access which fosters wider collaboration and increased citations

- maximum visibility for your research: over $100 \mathrm{M}$ website views per year

At BMC, research is always in progress.

Learn more biomedcentral.com/submission 